\title{
Differing Intentions and Behaviours about the HPV Vaccine among Midwestern Undergraduate Men and Women
}

\section{Theresa Hunter $^{*}$ and Melissa Weinstein}

Department of Applied Health Science, Indiana University School of Public Health, Bloomington, Indiana, USA

*Corresponding author: Theresa Hunter, Department of Applied Health Science, Indiana University School of Public Health, Bloomington, Indiana, USA, E-mail: tmhunter@indiana.edu

Received date: February 27, 2016; Accepted date: March 28, 2016; Published date: March 31, 2016

Copyright: @ 2016 Hunter T, et al. This is an open-access article distributed under the terms of the Creative Commons Attribution License, which permits unrestricted use, distribution, and reproduction in any medium, provided the original author and source are credited.

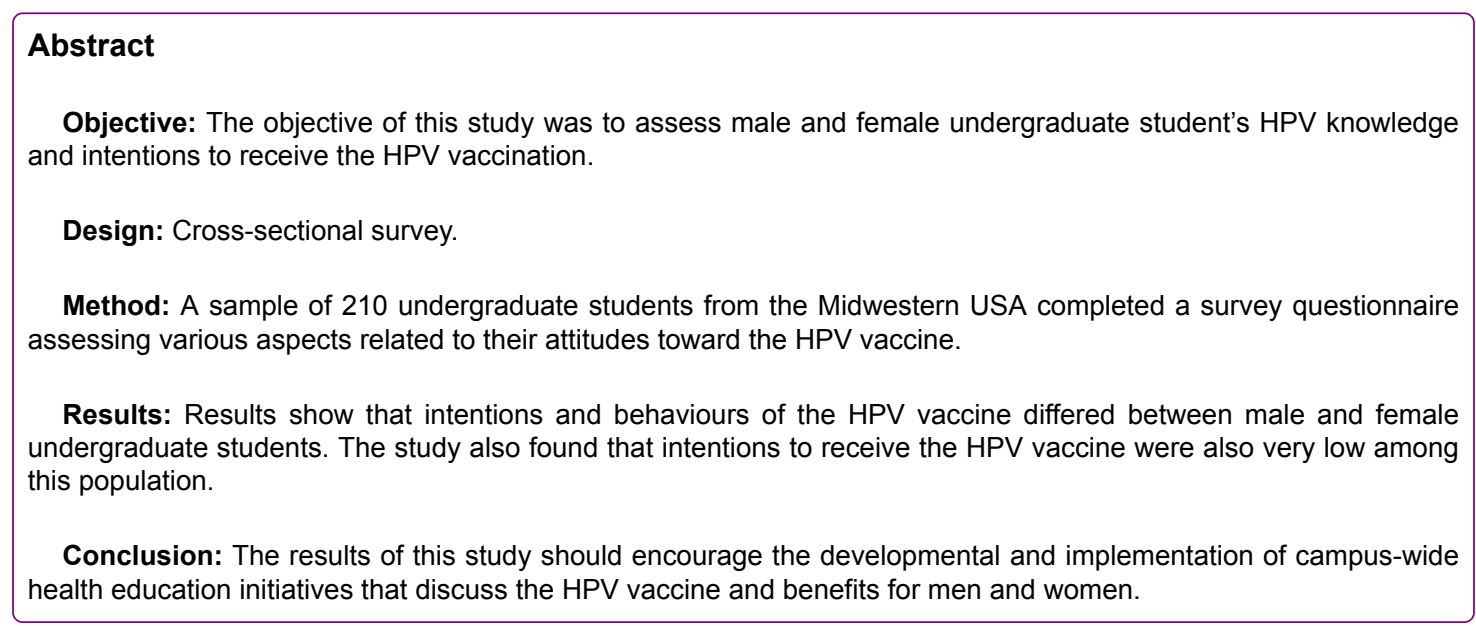

Keywords: HPV vaccination; Knowledge; Perceived benefits; Undergraduate students; USA

\section{Introduction}

The Human papillomavirus (HPV) is a major public health concern, especially for young adults. Human papillomavirus (HPV) is the most common sexually transmitted infection in the United States [1]. About 79 million Americans are currently infected with HPV and it is estimated that about 14 million people become newly infected each year [2]. HPV is a causative or contributing agent of genital warts and several types of cancers, such as cervical, vaginal, vulvar, anal, penile, and cancers of the head and neck $[1,3]$. In the USA, there are also about 17,000 women and 9,000 men affected by HPV-related cancers each year [4].

The U.S. Food and Drug Administration (FDA) approved a quadravalent HPV vaccine against types $6,11,16$, and 18 for use in women aged 9-26 years old in 2006 [3,5]. In 2009, the FDA approved Gardasil, a quadravalent HPV vaccine produced by Merck Pharmaceuticals, for use among men aged 9-26 years [3,6]. HPV vaccines are safe and the HPV vaccine has been proven to be effective in preventing HPV and related diseases in men [4]. When given in the recommended age groups, the HPV vaccine can protect men and women against diseases such as cancer [2].

While the HPV vaccine has been available for several years, HPV vaccination rates remain low [2]. HPV vaccination rates continue to lag behind rates for Tdap and quadrivalent meningococcal conjugate vaccines, even though the Advisory Committee on Immunization
Practices (ACIP) recommends that preteens (ages 11 or 12) get one dose of quadrivalent meningococcal conjugate vaccine, one dose of HPV vaccine, and one dose of Tdap vaccine during a single visit [6]. Despite vulnerability to HPV, $40 \%$ of adolescent girls and over $60 \%$ of adolescent boys have not started the HPV vaccine series [2]. This is very concerning since approximately half of the 14 million persons who are newly infected with HPV each year are between the ages of 15 to 24 years old [7].

In order to better understand the reasons why adolescents and young adults are not getting the HPV vaccination, it is vital to measure the knowledge and believes regarding the vaccine among this age group. In college, young people establish patterns of behaviour and make lifestyle choices that affect both their current and future health [8]. Thus, this period of emerging adulthood is a critical time for interventions on long-term health behaviour patterns.

To date, published studies have not compared HPV vaccine intention and behaviours among male and female Midwestern college students in the USA. The purpose of this study was to determine if there was a significant difference between HPV knowledge and vaccination intention among male and female undergraduate college students. Understanding this information can help health care providers, health educators, and public health professionals to better educate young adults on the importance HPV and the health implications that HPV could have on them. 


\section{Methods}

\section{Study sample}

A convenience sample $(\mathrm{n}=210)$ was recruited from a public undergraduate university in the Midwest region of the USA. Based on Cohen's primer to determine sample size, the estimated total sample size of $n=210$ was determined assuming power of $90 \%$ with $\alpha=0.05$ to determine a small effect for a multiple regression analysis. Participants were recruited from all undergraduate academic levels. Before the survey was administered, contact was made with various university lecturers who allowed recruitment of students from their classes. Students chose to complete the survey during class breaks and after class. Students were made fully aware that participating in the survey was completely voluntary and were informed that their responses would remain confidential. The study was approved by the Indiana University Institutional Review Board.

\section{Data collection}

A self-administered questionnaire was used in this study. The survey instrument included demographic factors such as age, ethnicity, race, and academic year. Items to assess perceived risk and severity, level of concern about HPV-related cancers (penile, anal, head and neck) and genital warts, vaccination intentions, and HPV knowledge were also included. The instrument format incorporated dichotomous, categorical, ordinal, and scale items as well as open-ended questions.

\section{Data analysis}

Data were analysed using SPSS statistical software version 22.0. This software was used for descriptive statistics where data is shown as percentages, means, and standard deviations to show demographic information. T-tests and chi-square tests were used to identify associations between male and female students' perceptions of the vaccination and perceived risk and perceived severity of HPV. Frequency distributions were used to test normality for each of these variables.

\section{Results}

\section{Demographic characteristics}

The sample consisted of 210 participants. A majority of participants identified as male (55.2\%). The ages of participants ranged from 18-26 years old. Age was divided into four groups: 18-19 (29.5\%), 20-21 (44.7\%), 22-23 (24.8\%), and 24-26 (1.0\%) years. The majority of respondents identified as White (71.9\%) followed by Asian (14.8\%), African American (7.1\%) and Native American or Pacific Islander (3.4\%). The majority of participants identified as non-Hispanic (97.2\%) and Hispanic (2.8\%). A full description of the demographic information is shown in Table 1.

\section{Vaccination intention and behaviour}

The majority of participants (59.0\%) indicated that they do not plan to receive the HPV vaccine and $20.9 \%$ of participants indicated that although they have considered receiving the vaccine, they have not yet been vaccinated (Table 2). Of the 210 participants in this study, only $20.0 \%(n=42)$ indicated that they already received the HPV vaccine. All participants that had received the HPV vaccine indicated that they were informed about this vaccination from a health care provider. All male and female participants that had received the HPV vaccine prior to the study indicated that they were informed about this vaccination from a health care provider.

\begin{tabular}{|c|c|c|}
\hline & $\mathbf{N}$ & $\begin{array}{l}\text { Percent of Total } \\
(\mathrm{N}=210)\end{array}$ \\
\hline \multicolumn{3}{|l|}{ Sex } \\
\hline Female & 94 & 44.8 \\
\hline Male & 116 & 55.2 \\
\hline \multicolumn{3}{|l|}{ Race/Ethnicity } \\
\hline White & 151 & 71.9 \\
\hline Asian & 31 & 14.8 \\
\hline African American & 15 & 7.1 \\
\hline Native American or Pacific Islander & 7 & 3.4 \\
\hline Hispanic & 6 & 2.8 \\
\hline \multicolumn{3}{|l|}{ Age (Years) } \\
\hline 18 & 16 & 7.6 \\
\hline 19 & 46 & 21.9 \\
\hline 20 & 51 & 24.3 \\
\hline 21 & 43 & 20.4 \\
\hline 22 & 38 & 18.1 \\
\hline 23 & 14 & 6.7 \\
\hline 24 & 1 & 0.5 \\
\hline 25 & 0 & 0 \\
\hline 26 & 1 & 0.5 \\
\hline
\end{tabular}

Table 1: Demographic Profile of Participants.

Males: Among the male participants, $80.2 \%$ indicated that they do not plan to receive the HPV vaccine. Although $13.8 \%$ of male participants indicated that they have considered receiving the HPV vaccination, they have not yet been vaccinated (Table 2). Of the 116 male participants in the study, only $6 \%$ indicated that they already received the HPV vaccine.

\begin{tabular}{|l|l|l|l|}
\hline & Males & Females & $\begin{array}{l}\text { Percent of } \\
\text { Total } \\
\text { (N=210) }\end{array}$ \\
\hline Do not plan to get vaccinated & 93 & 31 & 59 \\
\hline Considered getting vaccinated & 16 & 28 & 20.9 \\
\hline Have received the vaccination & 7 & 35 & 20 \\
\hline
\end{tabular}

Table 2: Vaccination Intention by Sex.

Females: Among the female participants, 32.9\% indicated that they do not plan to receive the HPV vaccine and $29.8 \%$ of female participants indicated that although they have considered receiving the vaccine, they have not yet been vaccinated (Table 2). Of the 94 female 
participants in the study, $37.2 \%$ indicated that they have already received the $\mathrm{HPV}$ vaccine.

\section{Awareness of the HPV vaccine}

Among total participants, $34.3 \%$ indicated that they had not heard of the HPV vaccine prior to the study. A majority (51\%) of male participants indicated that they were not aware of the HPV vaccine prior to the study, compared to only $14 \%$ of female participants indicating that they were not aware of the vaccine prior to the study.

\section{Discussion}

To date, the majority of HPV research has focused on the female population, without recognizing the infection as a concern for men. The present study is unique in how it compares vaccination intentions and behaviour among young men and women. This study provides potential insight into the low rate of males receiving the HPV vaccine.

While $65.7 \%$ of participants indicated that they have heard of the HPV vaccine, this awareness and knowledge of the vaccine differs for men and women. While $86 \%$ of female participants are aware that there is a vaccine for HPV, only $49 \%$ of male participants were aware that a vaccine exists. Furthermore, out of the male participants who have indicated that they know of the HPV vaccine, a majority of them were unaware that the vaccine was available for men, with $80 \%$ of male study participants revealing that they believed the HPV vaccine to only be an option for women. This may be a reflection of minimal attempts to date to increase HPV knowledge among male college students, particularly in the Midwest.

The current research has important implications for HPV awareness and educational initiatives. Study findings suggest that health education is needed to promote increased knowledge of the HPV vaccine among college students, especially male students. Given the low levels of awareness of the HPV vaccine observed among college students in the Midwest, increasing HPV education is an appropriate starting point.

Limitations of the current study should be considered when interpreting the results. When seeking information on lifestyle behaviours, the potential for bias always exists. Although the sample recruited for the study was diverse, the sample as a whole was relatively small. Moreover, as it was a convenience sample, findings may not generalize to Midwestern US undergraduate students. These results may also be constrained by the self-reporting nature of this questionnaire. Future studies should investigate college students' HPV vaccination intentions and behaviours utilizing a larger sample size.

\section{Conclusion}

While the female population is impacted by HPV at a slightly higher rate than the male population, prevention efforts need to target both groups. Findings from the present study suggest that awareness and knowledge of the HPV vaccine may increase HPV vaccination rates among young men and women. It is important to expand education efforts to improve upon understanding of the prevalence and consequences of HPV for both females and males, along with efforts to promote HPV prevention and care.

\section{References}

1. Palefsky JM (2010) Human papillomavirus-related disease in men: not just a women's health issue. Journal of Adolescent Health 46: S12-S19.

2. Centers for Disease Control and Prevention (2014) Genital HPV infection: CDC fact sheet. Sexually Transmitted Diseases.

3. Centers for Disease Control and Prevention (2010) FDA licensure of quadrivalent human papillomavirus vaccine (HPV4, Gardasil) for use in males and guidance from the Advisory Committee on Immunization Practices. Morbidity and Mortality Weekly Report 59: 630-632.

4. Giuliano AR, Palefsky JM, Goldstone S, Moreira ED, Penny ME, et al. (2011) Efficacy of quadrivalent HPV vaccine against HPV infection and disease in males. New England Journal of Medicine 364: 401-411.

5. Markowitz LE, Dunne EF, Saraiya M, Lawson HW, Chesson H, et al. (2007) Quadrivalent human papillomavirus vaccine: recommendations of the Advisory Committee on Immunization Practices (ACIP). Morbidity and Mortality Weekly Report 56: 1-24.

6. Baker CJ, Pickering LK, Chilton L, Cieslak P, Ehresmann KR, et al. (2010) Recommended adult immunization schedule: United States, 2010. Annals of Internal Medicine 152: 40-44.

7. Centers for Disease Control and Prevention (2015) Teen Vaccination Coverage: 2014 National Immunization Survey-Teen (NIS-Teen).

8. Nelson LJ (2005) Distinguishing features of emerging adulthood: the role of self-classification as an adult. Journal of Adolescent Research 20. 